used to quantify HAA-DNA adduct concentration and determine polymorphisms in genes involved in HAA metabolism and DNA repair.

Results In the preliminary data, HAA-DNA adducts were detectable in 17 of 23 individuals. Results show that dietary HAAs were predictive of adduct levels (Spearman Correlation Coefficient $=0.39$, $\mathrm{p}=0.06$ ). Further analyses on the remaining cohort will be conducted to model adduct levels as a function of dietary HAAs and other relevant dietary, lifestyle and genetic factors; gene-diet interactions will also be explored.

Conclusion This research aims to contribute to understanding the initial steps in this potentially carcinogenic pathway between meat consumption and cancer - important for assessing causality and the prevention of modifiable exposures.

Funding Supported by the Queen's University Terry Fox Foundation Training Program in Transdisciplinary Cancer Research in partnership with CIHR.

\section{P2-115 DEMAND AND CONTROL AT WORK AND BLOOD PRESSURE: SYSTEMATIC REVIEW AND META-ANALYSIS}

doi:10.1136/jech.2011.142976i.50

${ }^{1} Y$ H M Hökerberg, ${ }^{2} E$ Faerstein, ${ }^{1} \mathrm{C}$ A F de Andrade, ${ }^{1} \mathrm{R}$ Fernandes, ${ }^{1} \mathrm{~S}$ R L Passos. ${ }^{1}$ Evandro Chagas Clinical Research Institute, Oswaldo Cruz Foundation, Rio de Janeiro, RJ, Brazil; ${ }^{2}$ Institute of Social Medicine, University of the Rio de Janeiro State, Rio de Janeiro, RJ, Brazil

Karasek postulated that high job strain, an interaction between high psychological demands and low control at work, increases the risk of ill-health.

Objective Systematic review/meta-analysis of the association between job strain and blood pressure (BP).

Methods Target studies were published on Pubmed, Lilacs, SciELO, PsycInfo, Embase and Web of Science, until July 2009. Data extraction was conducted independently by 2 of 3 reviewers using a standardised form.

Results The search retrieved 1377 studies and 51 fulfilled the eligibility criteria, mostly cross-sectional and conducted in Europe or USA. Most of them applied the job content questionnaire $(89.4 \%)$ and used the Karasek's quadrant categories (78.4\%). Casual BP was measured in $26(50 \%)$, ambulatory BP monitored in 22 (84.6\%), selfmeasured BP in two (3.8\%) and self-reported hypertension in two studies (3.8\%). Hypertension was the outcome in 16 studies (30.8\%), 9 of them defined by BP $>140 / 90 \mathrm{~mm} \mathrm{Hg}$. High strain was associated with high BP/hypertension in $27 / 51$ (52.9\%) studies. Meta-analysis could be only performed for nine hypertension studies, for which the association was not confirmed neither for high strain ( $O R c=1.08,95 \%$ CI 0.98 to 1.19), high psychological demands $(\mathrm{OR} c=1.08,95 \%$ CI 0.98 to 1.19$)$ nor for high control $(\mathrm{OR} c=1.02$, $95 \%$ CI 0.94 to 1.11), with no evidence of publication bias.

Conclusion There is weak evidence in favour of the association between job strain and $\mathrm{BP} /$ hypertension. Comparisons were hampered by methods heterogeneity, particularly: inclusion criteria, data collection and exposure/outcome definition. Further research should include longitudinal design, low and middle-income countries and female workers, still lacking.

\section{P2-116 ADIPOSITY AND ITS CONTRIBUTION TO INDIVIDUAL AND REGIONAL DIFFERENCES IN BLOOD PRESSURE: THE KADOORIE BIOBANK STUDY OF 0.5 MILLION PEOPLE IN CHINA}

doi:10.1136/jech.2011.142976i.51

${ }^{1} \mathrm{~L}$ S Hong, ${ }^{* 2} \mathrm{~L}$ Li, ${ }^{1} \mathrm{M}$ Smith, ${ }^{1} \mathrm{~B}$ Lacey, ${ }^{2} Y$ Guo, ${ }^{1} \mathrm{~S}$ Lewington, ${ }^{1} \mathrm{G}$ Whitlock, ${ }^{1} \mathrm{R}$ Collins, ${ }^{3} \mathrm{~J}$ Chen, ${ }^{1} \mathrm{R}$ Peto, ${ }^{1} \mathrm{Z}$ Chen. ${ }^{1}$ Clinical Trial Service Unit \& Epidemiological Studies Unit
(CTSU), Nuffield Department of Clinical Medicine, University of Oxford, Oxford, UK; ${ }^{2}$ Chinese Academy of Medical Sciences, Dong Cheng District, Beijing, China; ${ }^{3}$ Chinese Centre for Disease Control and Prevention, Chang Ping District, Beijing, China

Introduction Raised blood pressure (BP) is a leading cause of premature death in China, but its determinants are not well understood. One likely determinant, adiposity, is increasing rapidly in much of China. We assessed the extent to which differences in BP can be accounted for by differences in body mass index (BMI) and in waist circumference (WC)

Methods We examined cross-sectional data from $>510000$ adults aged 30-79 recruited from five rural and five urban areas in China. Height, weight, systolic BP and WC were measured for all participants. Means and associations of SBP with age, BMI and WC were estimated in men and women separately.

Results The population means (SD) of SBP and BMI in men were $132(20) \mathrm{mm} \mathrm{Hg}$ and $23.4(3.2) \mathrm{kg} / \mathrm{m}^{2}$. Age-adjusted area means of SBP ranged from 126 to $136 \mathrm{~mm} \mathrm{Hg}(\mathrm{F}=77 \mathrm{p}<0.0001$, $\mathrm{R}^{2}=0.09$ ), and area means of BMI ranged from $22.0 \mathrm{~kg} / \mathrm{m}^{2}$ to $25.4 \mathrm{~kg} / \mathrm{m}^{2}$. Overall, and within each area, SBP was approximately linearly associated with BMI: overall $+17 \mathrm{~mm} \mathrm{Hg}$ SBP per $+10 \mathrm{~kg} / \mathrm{m}^{2}$ BMI. However, further adjusting SBP for BMI only accounted for an additional $7 \%$ of individual variation in SBP, and had little effect on differences between area means of SBP $(F=30 \mathrm{p}<0.0001)$. Despite lower BMI in rural vs urban areas ( 22.7 vs $24.3 \mathrm{~kg} / \mathrm{m}^{2}$ ), mean SBP was higher in rural areas (132 vs $131 \mathrm{~mm} \mathrm{Hg}, \mathrm{p}<0.0001)$. Similar patterns were found for WC, and in women.

Conclusions SBP is consistently associated with adiposity in individuals, but differences in adiposity do not account for most of the differences in SBP between individuals or between areas.

\section{P2-117 THE HYPERTRIGLYCERIDEMIC-WAIST PHENOTYPE AND WEIGHT GAIN IN CHILDHOOD}

doi:10.1136/jech.2011.142976i.52

B Horta, ${ }^{*}$ D Gigante, R Haack. Universidade Federal de Pelotas, Pelotas, Brazil

Evidence suggests that the hypertriglyceridemic-waist phenotype is related to the occurrence of cardiovascular metabolic risk factors. This study was aimed at assessing the relationship between weight in childhood and the presence of hypertriglyceridemic-waist phenotype. The 1982 Pelotas birth cohort included 5914 children who were born in three maternities in Pelotas, southern Brazil. The subjects have been followed-up for several times. In 2004-2005 (mean age 23 years), we attempted to trace the whole cohort and obtain blood samples. Conditional growth modelling was used to assess the association between the phenotype and weight gain from birth to 20, and from 20 to 42 months. Adjusted analyses controlled for household assets index, family income, maternal schooling at birth, maternal smoking during pregnancy, and breastfeeding duration. In 2004-2005, we interviewed 4297 subjects, and collected blood of 3911. Among small-for-gestational age subjects, weight gain in the first 20 months was not associated with the phenotype. But, those subjects whose weight gain from 20 to 42 months of age was faster than that predicted from birthweight and weight-for-age $z$-score at mean age of 20 months had a higher risk of presenting the phenotype [1.73 (95\% CI 1.15 to 2.79)]. Among subjects whose birthweight was adequate-for-gestational age weight gain in childhood increased the risk of having the phenotype. These findings suggest that among small-for-gestational age infants, early weight gain is not related to the presence of metabolic cardiovascular risk factors. 